\title{
Exploration and Implementation of Intelligent Park Information System based on Cloud Computing and Internet of Things
}

\author{
https://doi.org/10.3991/ijoe.v15i01.9783 \\ Mao Chen \\ Fuzhou Polytechnic of Information Technology, Fuzhou, China \\ Yong Lin $(\bowtie)$ \\ Fujian Business University, Fuzhou, China \\ yonglinsub381@126.com
}

\begin{abstract}
To effectively improve the operation, management, and service level of the intelligent park, a preliminary study and discussion is conducted on the design and implementation of the Internet of Things (IoT) information system in the intelligent park. Firstly, the hardware architecture and technical scheme of the information system of intelligent park based on Internet of things and cloud computing are proposed, and the information system architecture of the intelligent park is introduced. The construction of the system architecture is based on the three-tier architecture of perception, network and application; secondly, based on this system architecture, the functions of each component of the information system are explained from the perception layer, network layer and application layer, and some typical sensors in the system are introduced; finally, the system is analyzed experimentally. The results show that the system can realize the interconnection among various communication terminals and improve the efficiency of cooperation among various systems. It can be concluded that the information system of the intelligent park based on cloud computing and Internet of things can achieve the desired effect.
\end{abstract}

Keywords-Cloud computing, Internet of Things (IoT), intelligent park, information system

\section{Introduction}

At present, the technology of Internet of things (IoT) is still at the initial stage, and all kinds of technologies need to be developed and perfected. Information processing and analysis technology and level greatly determine the level of intelligent handling of the IoT. Current technologies include cloud computing, intelligent recognition and other advanced technologies. Among them, the cloud computing is especially important. It can be said to be the core of the realization of IoT, and it promotes the effective integration of IoT and Internet and further promotes the realization of the goal of intelligent earth. 
Paper-Exploration and Implementation of Intelligent Park Information System...

The goal of building intelligent ark is multifaceted, and it is mainly reflected in the following three aspects. First, the resources of the park can be effectively integrated through the construction of the park's wisdom. As a result, the resources of each unit in the park will be able to maximize its potential and avoid repeated investment in some of the resources that can be shared by the various units in the park, so as to realize the maximum benefit between the park and the enterprise. Second, through the construction of information technology in the park, the information of the park is displayed and published objectively and efficiently in real time and multi-platform, which makes the park information distributed according to the different distribution rules, such as units and personnel, and gradually achieves information distribution on demand. Third, through the establishment of the intelligent information platform of the park, the original independent system is gradually unified to an information platform, and the real-time effective connectivity and monitoring among the units is realized. In a word, through the construction of the intelligent park, some problems such as poor communication, duplication of resources and information island are solved to achieve the goal of win-win cooperation between the enterprises and the park.

Therefore, based on the information and intelligence of the intelligent park, the IoT and cloud computing technology are used to study and propose an effective solution to the information island. Through the intelligent construction of environmental monitoring, transportation, security and information fusion in the park, it provides reliable basic software and hardware, rich and diverse shared resources, and high-cost performance service and management.

\section{$2 \quad$ Literature Review}

At present, the construction and development of smart city is quite popular. Its construction and related fields involve all aspects of production and life in various fields and cities. Vanolo (2014) pointed out that the intelligent park is an important part and epitome of the smart city, and its propulsion and development model has a great effect on the construction and development of smart city [1]. Through the discovery of the advantages and disadvantages in the development and construction of the intelligent park, the development strategy of smart city can be revised in time, so that the urban intellectualization can be smoothly carried out, and the efficiency of the urban intelligent development can be improved. Hollands (2015) and Gabrys (2014) mentioned in the related literature that, by the end of May 2014, 222 cities have been built in the country, and a total of 293 cities at the county level and above have been approved [2,3]. The construction of smart city involves more than 20 fields, including information infrastructure, e-government, social security, intelligent transportation and intelligent medical treatment. At the end of 2014, 25 ministries and commissions, including the National Development and Reform Commission, the Ministry of Industry, the Ministry of Science and Technology and the Ministry of Public Security, jointly supported the construction and planning of smart cities. Komninos et al. (2014) mentioned in the related literature that the National Development and Reform 
Paper-Exploration and Implementation of Intelligent Park Information System...

Commission interpreted the intelligent city as the advanced city of urbanization and information. The specific contents include: a large number of IoT related technologies, cloud computing technology, graphic image technology, satellite navigation technology, and intelligent processing technology. The management and service, and the planning are intelligently constructed, gradually realizing the city information "upgrade" process, effectively improving the level of management, the people's living standards, and the development of various industries [4].

In the development process of smart city, intelligent park is an indispensable part of it. The healthy development of intelligent park can effectively provide reference for the development of smart city. Gaur et al. (2015) believed that the intelligent park is only a relatively small area for the city, but it is the pioneer of the process of urban intelligence, which can effectively reflect the correctness, rationality, and efficiency of the strategy of intelligent construction [5]. Anthopoulos and Fitsilis (2014) mentioned that the characteristics of intelligent park can be summed up as three words: perception, interconnection and intelligence. Perception: in the intelligent park, through the application of the advanced technology related to IoT, the state of the important management objects in the various systems of the park can get the correct perception and measurement [6]. Interconnection: in the intelligent park, through high-speed communication and network, the relatively independent systems and departments can achieve efficient interconnection, enhance communication efficiency, and form an efficient ecological structure of the park. Intelligence: in the intelligent park, the intelligent autonomous management of the park is realized through the construction of various intelligent application systems. Based on the technologies of cloud computing and business intelligence processing, the effective multi-directional, multi-angle and comprehensive analysis, judgment and prediction are implemented by integrating and collaborating the information from the sources, thus promoting intelligent decision-making of management at home and abroad. Crivello (2014) and Menouar et al. (2017) believed that, at present, the construction of intelligent parks needs further study in four aspects, namely, design limitations, fragmentation of information, building hollowing out, and security vulnerability [7,8].

This paper is a common problem in the actual construction of the intelligent park, that is, the lack of unified planning, the repeated construction of campus and enterprise information, thus forming a single information island. Relying on the 54 scientific and technological transformation platforms, based on the specific application of the new generation of information technologies, such as IoT, cloud computing, and big data, the solution of intelligent information system is put forward to make the real-time perception, more extensive interconnection and more intelligent analysis possible. This scheme not only provides an effective means to solve the increasingly complex information island disease, but also provides an efficient information intellectualization ability for improving the precision management and public service level of the park, and creating an intelligent and livable urban environment. 
Paper-Exploration and Implementation of Intelligent Park Information System...

\section{$3 \quad$ Method}

\subsection{Cloud computing}

Cloud computing can provide strong technical support for IoT, and its key technologies are:

Virtualization: As the most important technical basis for cloud computing, virtualization actually means that the unit of computing runs on a virtual basis rather than a real hardware base. Through the use of virtualization technology, the existing resources of the enterprise can be most reasonably configured and used. At the same time, the enterprises can allocate the resources according to their needs in time to achieve dynamic equilibrium in accordance with the changes of their business needs. Because of the realization of the hardware independence, this brings the system reliability effectively improved. In practical cloud computing applications, cloud computing services are implemented by computing virtualization, and corresponding applications are provided. At present, the application of virtualization technology in the central processing unit, operating system (OS), and server greatly improves the efficiency and quality of service.

Distributed file system: The search service provided by Google, facing the global users, because of its large user scale, has proposed a distributed processing technology and realized the collaborative work of millions of ordinary computers by using the distributed architecture. The distributed storage of massive data is realized through distributed file system, and the storage of massive data is realized through distributed database. DFS is the abbreviation of distributed file system. The file system was originally designed to provide services for local data in the local area network (LAN). DFS extends its services to the entire network. Sending samples can not only change the way of data storage and management, but also has the advantages of data backup and data security that the local file system does not have. The following is the typical application mode of DFS.

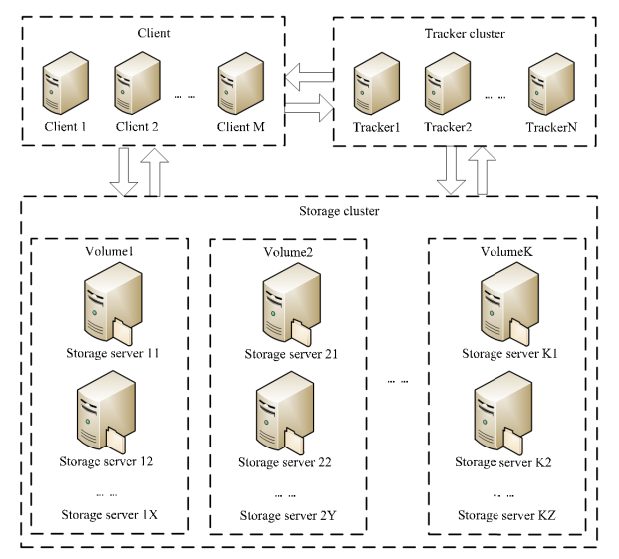

Fig. 1. Distributed file system 
Paper-Exploration and Implementation of Intelligent Park Information System...

DFS is a version of AFS as part of OSF's DCE (i.e. distributed computing environment) file system. If access to files is limited to one user, DFS will be very easily implemented. Unfortunately, in reality, the restrictions on sending samples in many network environments are not realistic, so concurrency control is required to achieve access to multiple users.

Parallel computing: Parallel computing is a relatively simple preliminary idea: several computers can be able to achieve several times the ability of a single computer and reduce processing time to a fraction of that of a single computer. Thus, it can be seen that this is an ideal state. Information exchange and synchronization are usually required. Even so, the processing performance can still be effectively improved. Usually, parallel computing is divided into time parallel and spatial parallel computing. Time parallel computing usually refers to pipelining technology, whereas spatial parallel is calculated by parallel processing of many processors.

Cloud security: Because its model is the Internet-based computing model, the related services provided by cloud computing inevitably involve information security issues, such as information disclosure, illegal theft, virus attacks, and security vulnerabilities. The current cloud security has developed to the third generation of trusted cloud security. It is characterized by automatic security detection and automatic defense on Internet. The client can be configured and optimized to very small, thereby improving the related performance and reducing resource consumption.

Cloud service platform architecture: The information cloud service platform structure of intelligent park mainly consists of five levels, namely, IaaS layer, PaaS layer, SaaS layer, operation management layer, and user layer.

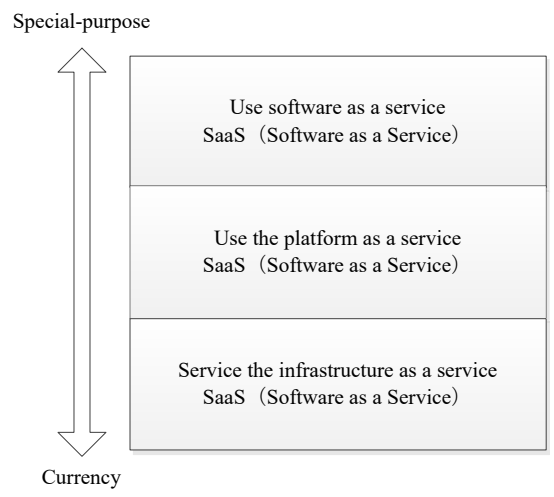

Fig. 2. Cloud computing service type

In addition, in recent years, there have been some OS based on cloud computing. For example, Windows Azure is an OS based on cloud computing released by Microsoft. The main purpose of the OS is to provide a platform for software developers to help develop applications that can run on cloud servers, data centers, Web, and personal computers. This service platform enables software developers to 
Paper-Exploration and Implementation of Intelligent Park Information System...

effectively utilize the tools and technologies that they are familiar with, and fully enhance the flexibility of various application development.

\subsection{Information system architecture of intelligent park}

The information system architecture of the intelligent park is based on the framework of ITU (International Telecommunication Union). The technical framework of the IoT includes the perception layer technology, the network layer technology and the application layer technology. The system architecture is based on three levels of perception, network and application, and realizes interconnection and resource sharing under unified standards. Through the deployment and setting of various types of sensors, the information in the park is perceived quickly and multifaceted. The information is transmitted through the transmission network to the application system, and the related information is generated and processed through various application systems. The system architecture is shown in Figure 3.

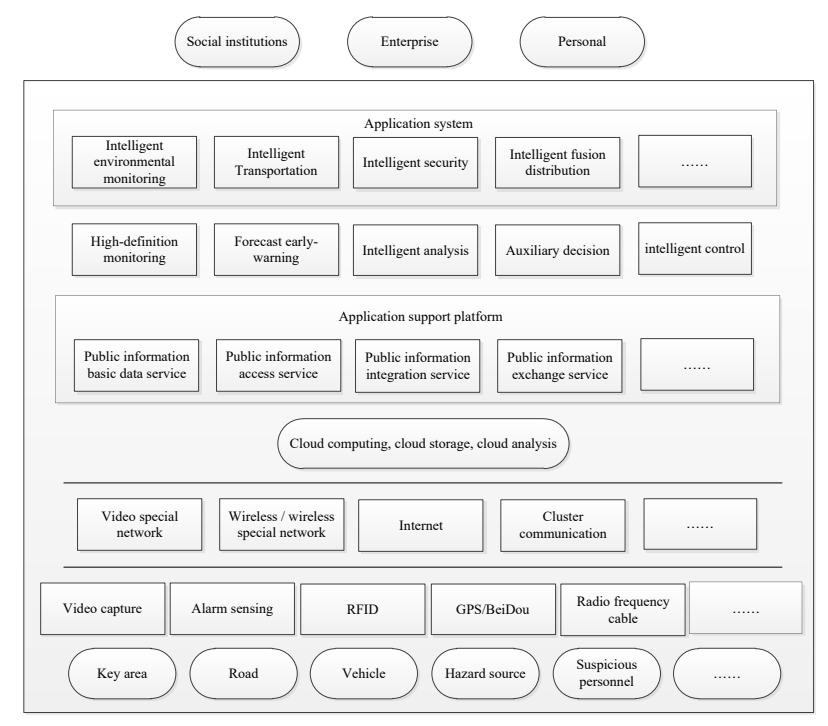

Fig. 3. Information system architecture of intelligent park

Perception is the basic data collection layer of safe city construction. Highdefinition video surveillance camera is used as the main base image acquisition unit, and the front monitoring scene is monitored in real time. Video recognition and behavior analysis technology are used to collect road vehicle information and illegal information. Ration frequency identification (RFID) technology and global positioning system (GPS) / BeiDou technology are widely used to collect real-time position and other relevant information of vehicles, personnel and articles, as well as the collection of other social security information. It is the largest part of the construction project of secure city. The wider the coverage of point placement is, the 
Paper-Exploration and Implementation of Intelligent Park Information System...

better the monitoring effect is. And the selection of equipment suitable for monitoring the scene is the key.

Network: network transport layer uses the advanced wireless broadband technology, optical fiber communication, and $3 \mathrm{G}$ wireless communication technology for covering the data acquisition location comprehensively, which needs to meet the bandwidth requirements.

Application: it is an important link in the full play of the system function. The cloud computing, cloud storage and cloud analysis technology are used to integrate the monitoring resources in the park, excavate the "massive data", and provide a full range of data support for the related applications. It is the result of the construction of the park's information construction, which provides the humanized and intelligent application interface for each park unit and district staff based on global information system (GIS) integrated management platform. It also provides data sharing service for park public security management, traffic management, emergency handling and command and dispatch.

\subsection{Application system design}

Intelligent environment monitoring and design: The real-time monitoring of environmental information (temperature, humidity and harmful gas content) in the park is realized through the environment monitoring unit and module (smoke and harmful gas) set in the park, and it is collected to the application support platform through the transmission network.

The environmental monitoring system includes two parts: environmental data acquisition and environment related data receiving and processing. Among them, the environmental data acquisition part contains sensors and wireless transmission two parts. The data receiving and processing part includes wireless data receiving and processing. The sensors of each node deployed in the park are sent from the transmission system to the environmental data processing section after the collection of environmental data. Through the data processing and analysis in the environmental data processing section, it will automatically trigger the related operations if the environment data is beyond the security range. Then, the transmission system will send the alarm information to the relevant managers and systems. The video can be viewed by calling the corresponding camera through the correlation relation that has been recorded in advance.

ZigBee technology is mature and widely used, which provides a new way of thinking for wireless monitoring. In some occasions, data exchange amount is not very large, and data format is relatively simple. ZigBee technology is very suitable for this occasion. Under normal conditions, ZigBee wireless sensor network consists of several components: processing computer, signal collector and acquisition terminal. The acquisition terminal is used to collect the wireless data and as nodes, it is responsible for sensing and processing the data. In the distributed environment, the signal collector is responsible for the radio frequency signals sent by each node, and sends the information to the processing computer through the RS-232 serial port, so as to implement the data storage and processing on the processing computer. The 
schematic diagram of intelligent environmental monitoring system is shown in Figure 4.

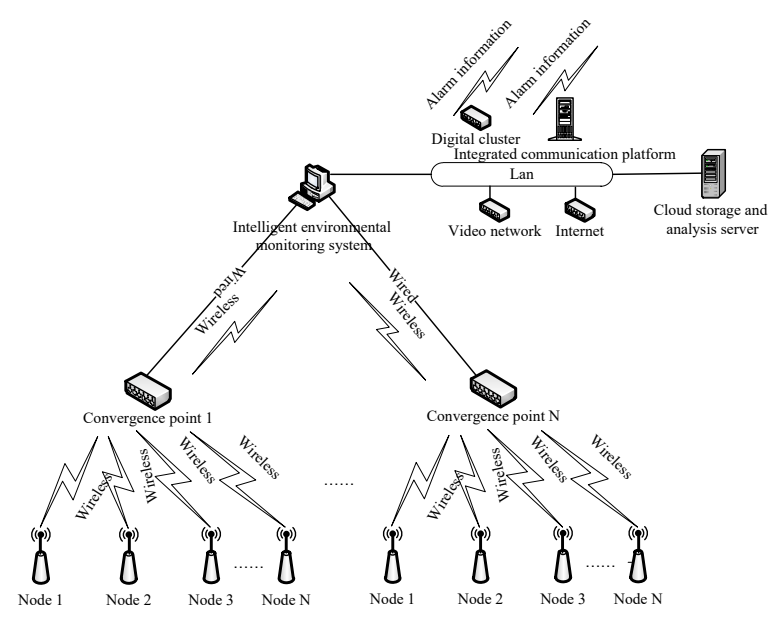

Fig. 4. Schematic diagram of intelligent environmental monitoring system

Through the deployment of multiple monitoring nodes, the collection of environmental data is realized. The collected data is sent to the gathering node through the wireless ZigBee technology, and then the data is analyzed, processed and stored through the wired or wireless network. The analysis results are used in conjunction with video network, and the analysis results are sent to the personnel of the management of the park, and the efficiency of monitoring and the response rate of the personnel are improved.

Intelligent traffic design: Through the integrated GPS/BeiDou module and RFID module in the vehicles in the park, the real-time monitoring of the location of each park in the park is made, the real-time situation of each section of the park is generated, and the support platform is collected through the transmission network. After the certification, the park staff can call real-time information of each link, and then choose the most suitable route or site for vehicle related operations. The certified vehicles in the park can be transferred from the park by RFID. The schematic diagram of intelligent transportation system is shown in Figure 5. 


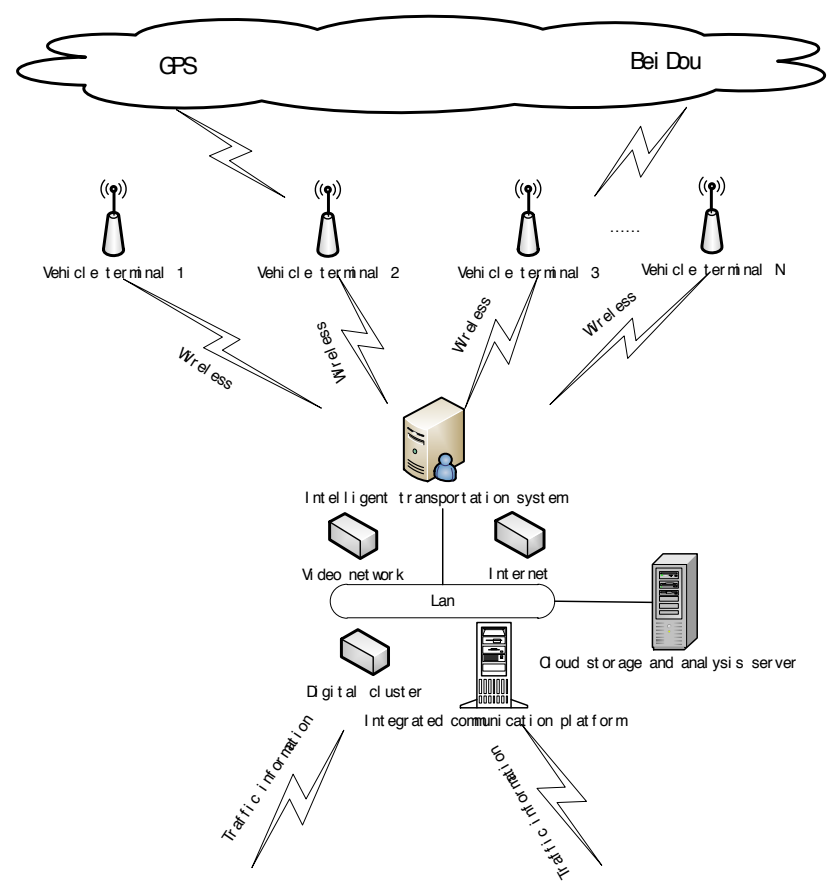

Fig. 5. Schematic diagram of intelligent transportation system

The GPS/BeiDou module integrated in the vehicles in the park can realize realtime monitoring of vehicles in the park and use video network. When traffic congestion occurs in the park, the congestion information is sent to the people who is responsible for the park management by digital cluster and integrated communication platform, so that the congestion of the park can be effectively processed.

Intelligent security design: Intelligent security refers to the video monitor in the surrounding area of the park and the key area to monitor all the sections, buildings and plants in the park in real time. By displaying the distribution of all the video resources on the GIS map, the operator can quickly open the video image by the way of the mouse selection, so that the video control on the GIS map is achieved. Based on a large number of video behavior analysis, the utilization and reliability of the system are improved. On the basis of video control, the intelligent security makes use of the advantages of large amount of data and wide analysis of cloud analysis in the cloud storage platform. It makes comprehensive use of video resources, and realizes intelligent analysis of personnel intensive alarm in the park, the warning of illegal stay at the boundary of the park, and the warning of suspicious items in specific areas. The schematic diagram of intelligent security system is shown in Figure 6. 


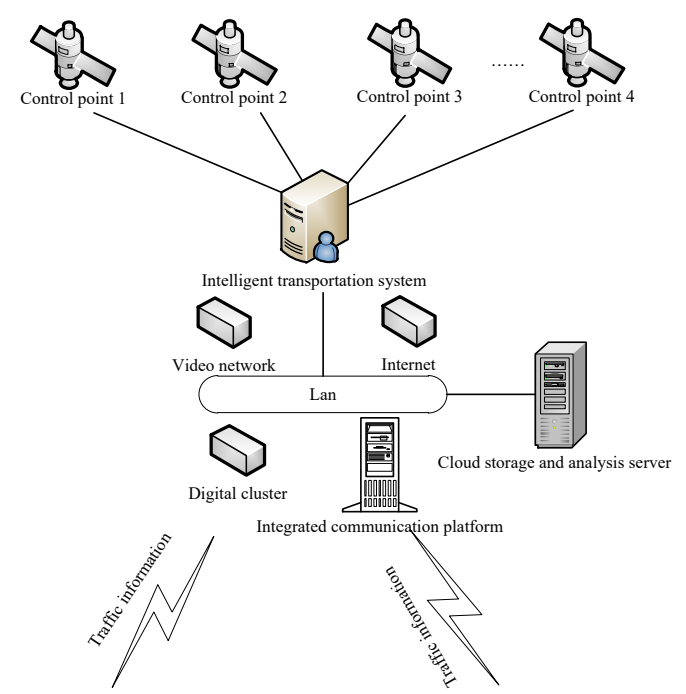

Fig. 6. Schematic diagram of intelligent security system

Intelligent fusion design: Receive and integrate the alarm information (such as toxic gas leak alarm) from the intelligent environmental monitoring system, traffic accidents sent from intelligent transportation system, and illegal intrusion information sent from intelligent security system. Through communication with the guard of the park, take the corresponding measures. By setting up the intelligent fusion system in the management of the park, the fusion of multi-angle and multi-directional park information is realized, which makes the generated information more comprehensive and timely. Through the interaction with the related units in the park, the municipal police system, the hospital and other departments, the sudden events can be handled quickly and effectively, and the effect of minimizing the accident harm can be achieved, which solves the phenomenon of information island in the subsystem. The schematic diagram of intelligent fusion is shown in Figure 7.

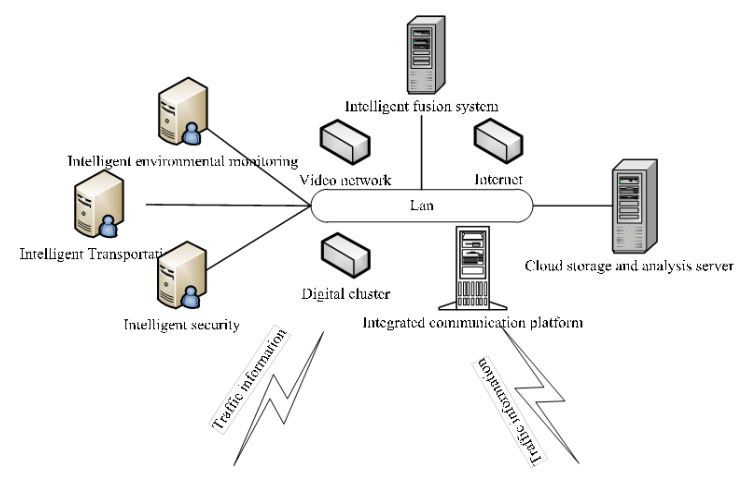

Fig. 7. Schematic diagram of intelligent fusion 
Paper-Exploration and Implementation of Intelligent Park Information System...

Application support platform: Using the means of cloud computing, cloud storage and cloud analysis, the support platform provides public basic data information services, public information wireless, wired access services, public integrated information services, and public information exchange services, to realize high-definition monitoring, prediction, early warning, intelligent analysis, auxiliary decision-making, and intelligent control.

\section{$4 \quad$ Results}

\subsection{Composition of the experimental network}

Equipment selection: Select the video server based on cloud computing to achieve effective monitoring of abnormal behavior in the park.

The AcroTetra digital trunked single carrier base station SB421 is selected as the outdoor base station, as shown in Figure 8.

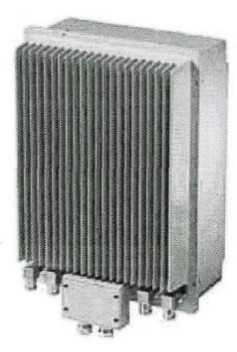

Fig. 8. Single carrier base station SB421

Select SepuraSRH3800 and integrate the world's leading GPS module, which can more easily and quickly position than the general GPS receiver even in the buildings. The location sensitivity reaches $-152 \mathrm{dbm}$, and the tracking sensitivity reaches $155 \mathrm{dbm}$. Through ETS300019Drop\&Vibration drop and vibration test, the waterproof and dust-proof can reach IEC529IP54 standard, the storage temperature range is -40 DEG $\mathrm{C}$ to $+85 \mathrm{DEG} \mathrm{C}$, and the environment temperature is $-20 \mathrm{DEG} \mathrm{C}$ to +60 DEG C, which can meet the storage and use of the North low temperature area.

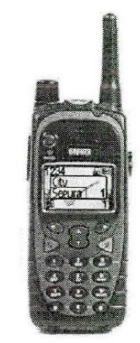

Fig. 9. SRH3800 handheld terminal 
Paper-Exploration and Implementation of Intelligent Park Information System...

The selected AcroUniteU7200 integrated communication platform is a new type of communication system. A variety of communication networks of wired communication and wireless communications, and analog and digital communications, can be integrated into a seamless network to achieve interconnection among various networks. Thus, the unified command and dispatch can be realized. The platform is shown in Figure 10.

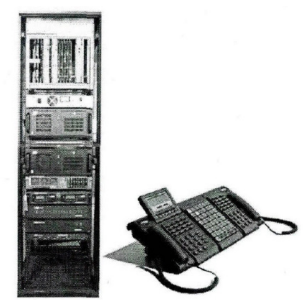

Fig. 10. AcroUnitedU7200 integrated communication platform

Second, the composition of the experimental network: the experimental network is composed of a LAN environment, access to video cloud storage and analysis server. Access an analog control point (control point 1), and select four computers with intelligent environment monitoring system software, intelligent transportation system software, intelligent security system software, and intelligent fusion system.

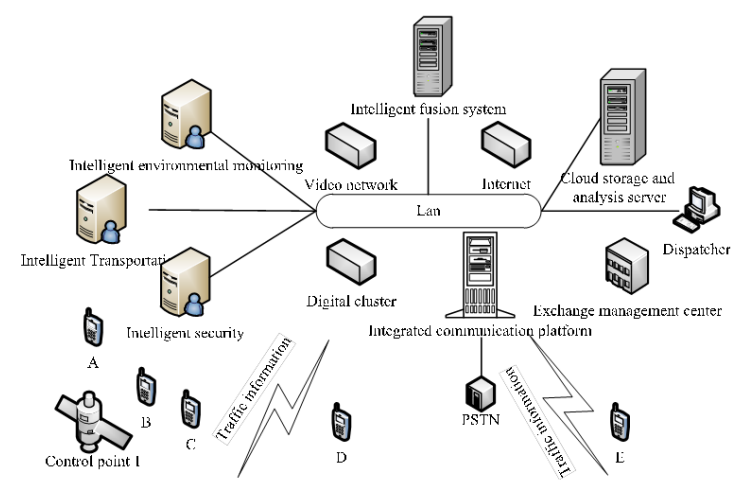

Fig. 11. Composition of experimental network

Configure AcroUniteU7200 integrated communication platform, switching management and dispatching desk. Configure 1 AcroTetra base station and three Sepura SRH3800 digital cluster handheld terminals (intelligent traffic - A, intelligent environmental monitoring - B, and intelligent security system - C) for park information system use, a fixed phone and a Sepura SRH3800 digital cluster handheld terminals D (intelligent fusion handler use), and a mobile phone E (park manager personnel use). 
Paper-Exploration and Implementation of Intelligent Park Information System...

\subsection{Intelligence interconnected park information system test}

The content of the test:

Cluster terminal positioning display: The three Sepura SRH3800 digital cluster handheld terminals are numbered $\mathrm{A}, \mathrm{B}$ and $\mathrm{C}$, respectively. In the smart security system, the location map is configured to receive location information sent from handheld terminals $\mathrm{A}, \mathrm{B}$ and $\mathrm{C}$.

Personnel gather alarm and fusion processing and distribution: In the smart security seats, delineation of the monitoring area and the establishment of personnel density upper limit is 15 . When the number of personnel in the control point is more than 15 , the intelligent security system gives warning hints. The traffic information and environmental information of the point of control are obtained, and the integrated information is generated after fusion processing and distributed to the terminals held by the relevant personnel.

PSTN is interconnected with a cluster handheld terminals and mobile phones. PSTN interconnects with digital cluster handheld terminals A, B and C through dial up of dispatching stations. The cluster D interacts with the digital cluster terminal A, $\mathrm{B}$ and $\mathrm{C}$, and cluster D realizes interworking with mobile $\mathrm{E}$.

The test results:

Digital cluster handheld terminal positioning display: In the intelligent security seat, the location information of $\mathrm{A}, \mathrm{B}, \mathrm{C}$ and $\mathrm{D}$ of the digital cluster handheld terminal is received and displayed on the map in real time, and the status is displayed normally and correctly.

Personnel gather alarm and fusion processing and distribution: When the number of people in the video is more than 15 , the intelligent security system automatically gives alarm and reports the alarm information (location and number of people over the limit) to the intelligent fusion system. After the intelligent transportation system and the intelligent environment monitoring system call the traffic and environmental information of the relevant location, the fusion is conducted to generate the integrated information and sent to the digital cluster hand-held terminals (intelligent traffic - A, intelligent environment monitoring - B, intelligent security system - C), and digital cluster handheld terminal D (intelligent fusion processing personnel use) and mobile E users (park manager personnel use).

PSTN is interconnected with cluster handheld terminals to implement scheduling. PSTN and digital cluster handheld terminal A, B and C realize interoperability through the dial-up of the dispatching station, and the voice is clear. The cluster $\mathrm{D}$ is interconnected with digital cluster terminal A, B, and $\mathrm{C}$ to achieve interoperability and the voice is clear. Cluster $\mathrm{D}$ is interconnected with mobile $\mathrm{E}$ to achieve interoperability and the voice is clear, so as to achieve timely and effective communication and processing of emergencies.

\section{Conclusion}

First of all, the hardware architecture and technical scheme of the intelligent park information system based on IoT and cloud computing is proposed. The information 
Paper-Exploration and Implementation of Intelligent Park Information System...

system architecture of the intelligent park is introduced. The system architecture is constructed based on the three layers of perception, network and application. Secondly, based on the system architecture, the functions of the information system are described from the perception layer, the network layer and the application layer, and some typical sensors used in the system are introduced. Finally, the system is experimentally analyzed. The results show that the system can realize the interconnection and communication among various communication terminals and improve the efficiency among the systems.

\section{References}

[1] Vanolo, A. (2014). Smartmentality: The Smart City as Disciplinary Strategy. Urban Studies, 51(5): 883-898. https://doi.org/10.1177/0042098013494427

[2] Hollands, R.G. (2015). Critical interventions into the corporate smart city. Cambridge Journal of Regions Economy \& Society, 8(1): 61-77. https://doi.org/10.1093/cjres/rsu011

[3] Gabrys, J. (2014). Programming Environments: Environmentality and Citizen Sensing in the Smart City. Environment \& Planning D Society \& Space, 32(1): 30-48. https://doi.org/10.1068/d16812

[4] Komninos, N, Bratsas, C, Kakderi, C. (2015). Smart city ontologies: Improving the effectiveness of smart city applications. 1(1): 1-16.

[5] Gaur, A., Scotney, B., Parr, G. (2015). Smart City Architecture and its Applications Based on IoT. Procedia Computer Science, 52(1): 1089-1094. https://doi.org/10.1016/j. procs.2015.05.122

[6] Anthopoulos, L.G., Fitsilis, P. (2014). Smart Cities and Their Roles in City Competition: A Classification. International Journal of Electronic Government Research, 10(1): 63-77. https://doi.org/10.4018/ijegr.2014010105

[7] Crivello, S. (2015). Urban Policy Mobilities: The Case of Turin as a Smart City. European Planning Studies, 23(5): 909-921. https://doi.org/10.1080/09654313.2014.891568

[8] Menouar, H, Guvenc, I, Akkaya, K. (2017). UAV-Enabled Intelligent Transportation Systems for the Smart City: Applications and Challenges. IEEE Communications Magazine, 55(3): 22-28. https://doi.org/10.1109/MCOM.2017.1600238CM

\section{$7 \quad$ Author}

Mao Chen is a Researcher of Fuzhou Institute of Technology College, Fuzhou Fujian, 350000, China. His research interests include Cloud Computing.

Yong Lin is a Researcher of Fujian Business University, Fuzhou Fujian, 350000, China. His research interests include Intelligent Park Information System.

Article submitted 30 October 2018. Resubmitted 20 November 2018. Final acceptance 12 December 2018. Final version published as submitted by the authors. 